\title{
THE MISSING X-RAY BACKGROUND
}

\author{
ANDREA COMASTRI \\ INAF-Osservatorio Astronomico di Bologna, \\ via Ranzani 1, I-40127 Bologna, Italy \\ E-mail: andrea.comastri@bo.astro.it
}

\begin{abstract}
The fraction of the hard X-ray background (XRB) resolved into individual sources by the deep Chandra and XMM-Newton surveys strongly depends on the adopted energy range and decreases with increasing energy. As a consequence the nature of the sources of the even harder $(>10 \mathrm{keV})$ XRB remains observationally poorly constrained. I will briefly discuss the need for X-ray observations above $10 \mathrm{keV}$.
\end{abstract}

\section{Introduction}

After the impressive achievements obtained by Chandra and XMM-Newton in terms of angular resolution and high energy throughput, almost all the papers dealing with X-ray observations of extragalactic sources begin with the following general statement: ... Deep Chandra and XMM-Newton surveys have resolved most of the XRB into discrete sources... While there are no doubts about the origin of the XRB, the above statement is true only when referred to the $2-10 \mathrm{keV}$ energy range and it is even more true around 2-3 $\mathrm{keV}$ rather than above $7-8 \mathrm{keV}$, where the resolved fraction is no more than $50 \%{ }^{22}$. At energies greater than $10 \mathrm{keV}$, where the bulk of the XRB energy density is produced, the resolved fraction is negligible, being strongly limited by the lack of imaging X-ray observations at high energies. As far as the $10-100 \mathrm{keV}$ band is concerned, we are currently facing the problem already encountered for the $2-10 \mathrm{keV}$ band right after a significant fraction of the $1 \mathrm{keV}$ background was resolved by Einstein ${ }^{8}$ surveys.

The most important difference is that a solid model for the XRB, based on the AGN unification scheme, is now available. First proposed by Setti and Woltjer ${ }^{18}$ and elaborated with an increasing level of details since that time 1339 the XRB synthesis is obtained assuming a dominant contribution of obscured AGN with a wide range of column densities and luminosities. Though differing in several details regarding the luminosity function and $\mathrm{X}$-ray spectral shape parameterization, the different flavours of synthesis 


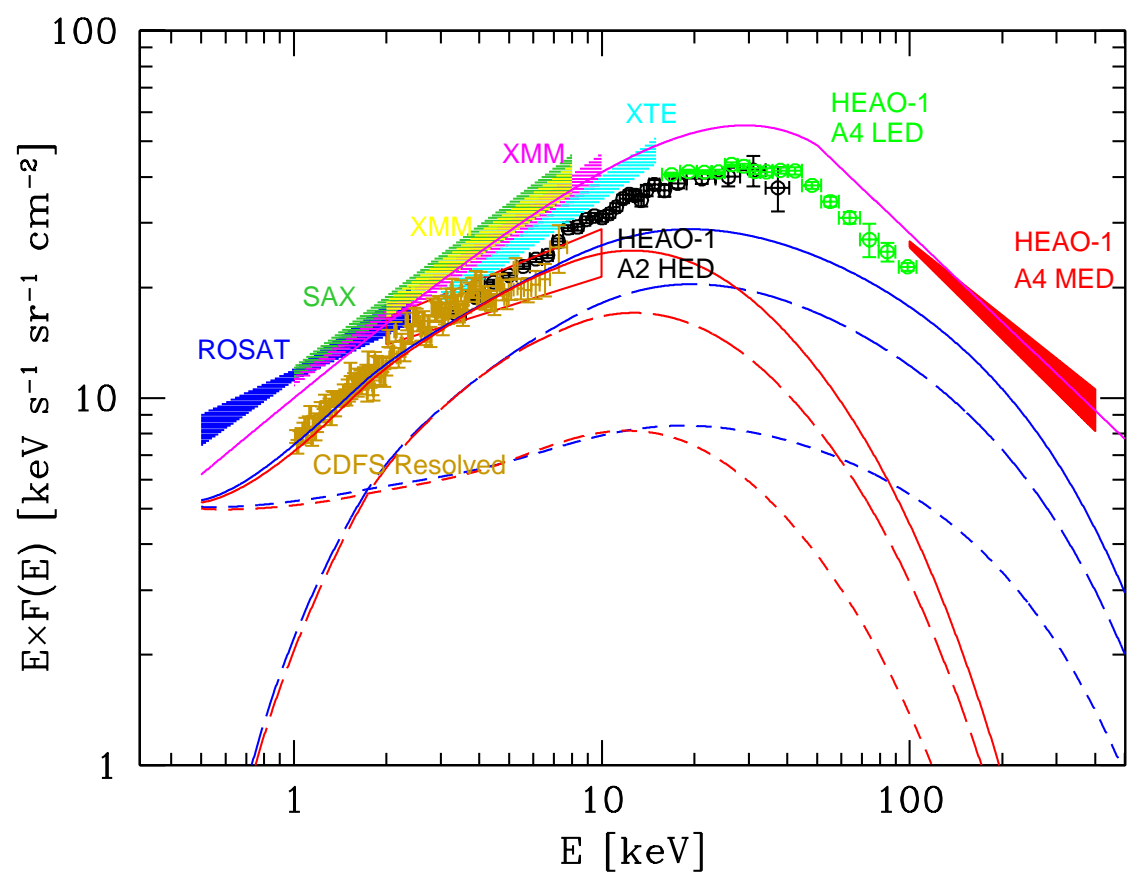

Figure 1. A selection of XRB spectral measurements collected from observations with different satellites as labeled. ROSAT blue ${ }^{7}$; BeppoSAX green ${ }^{21}$; XMM-Newton magenta 12 and yellow ${ }^{5}$; RXTE cyan ${ }^{15}$; HEAO1-A2 black ${ }^{10}$ and HEAO1-A4 LED green ${ }^{10}$; HEAO1-A4 MED red ${ }^{11}$. Also reported is the integrated contribution of resolved sources in the Chandra CDFS ${ }^{19}$ (gold points) and in XMM-Newton Lockman hole ${ }^{22}$ (red bowtie). The solid magenta curve represents the analytical fit of Gruber ${ }^{10}$ renormalized upward by $30 \%$ in order to fit the most recent measurements. The blue $\left(E_{\text {cut }}=400\right.$ $\mathrm{keV})$ and red $\left(E_{\text {cut }}=100 \mathrm{keV}\right)$ solid curves represent the integrated contribution of the model described in the text. The short-dashed curves correspond to unabsorbed AGN $\left(\log N_{H}<22 \mathrm{~cm}^{-2}\right)$, while the long-dashed curves correspond to obscured Comptonthin sources $\left(22<\log N_{H}<24 \mathrm{~cm}^{-2}\right)$.

models, published so far, share the same key feature: the X-ray obscuration. In the following I will refer to these as absorption models. 
Massive campaigns of multiwavelength follow-up observations have made possible to obtain spectroscopic and photometric redshifts for several hundreds of hard X-ray (2-10 keV) selected Chandra and XMM-Newton sources ${ }^{117}$ (see Tables 1 and 2 in Brandt et al. ${ }^{2}$ for a summary). The discovery of a sizable fraction of $\mathrm{X}$-ray obscured sources agrees, at least to a first approximation, with the absorption models predictions. However the observed absorption and redshift distributions are poorly reproduced by current models.

Although a rather obvious way to cope with these problems is to construct absorption models with different luminosity and absorption functions until a better agreement with the overall observational constraints is achieved (see for instance Ueda et al. ${ }^{20}$ ), a relatively large region of the parameter space remains unaccessible due to the lack of observational constraints.

Within the framework of absorption models, the shape of the XRB spectrum and intensity in the $10-100 \mathrm{keV}$ range, where most of its energy density is contained, is modeled assuming an important contribution from heavily obscured Compton thick $\left(N_{H}>1.5 \times 10^{24} \mathrm{~cm}^{-2}\right)$ sources around 20-30 keV. Moreover a high energy cut-off $\left(E_{c u t}\right)$ usually parameterized as an exponential roll-over with an e-folding energy of the order of a few hundreds of $\mathrm{keV}$ has to be present in the high energy spectrum of all the sources in order not to overproduce the observed XRB above $100 \mathrm{keV}$.

The most reliable observational constraints on the Compton thick AGN space density and the exponential cut-off energy have been obtained thanks to the PDS instrument on board BeppoSAX. Though limited to bright 10$100 \mathrm{keV}$ fluxes, the BeppoSAX results indicate that a fraction as high as $50 \%$ of the Seyfert 2 galaxies in the nearby Universe are obscured by Compton thick gas ${ }^{16}$, while the e-folding energy in the exponential cut-off spans a range from about 80 to more than $300 \mathrm{keV}^{14}$.

In the following I will briefly discuss the impact that different assumptions on the fraction of Compton thick sources and $E_{c u t}$ values of the continuum have on the synthesis of the high energy XRB spectrum. Given that the emission processes reponsible of Compton thick absorption and high energy cut-off are basically driven by Compton scattering, I will refer to this approach as Compton models. 


\section{Resolved and Unresoved XRB}

For the purposes of the present exercise I will adopt the parameters used by Comastri et al. (1995). Although there are compelling evidences which indicate that the evolution of the $\mathrm{X}$-ray luminosity function could not be parameterized by pure luminosity evolution anymore simple as postulated in the simplest versions of absorption models, ${ }^{20}$ it is important to point out that the most recent findings are not expected to substantially modify the model predictions above $10 \mathrm{keV}$.

The starting and admittely extreme assumption is that Compton thick absorption is not energetically relevant. Since the observational constraints on the presence of Compton thick sources beyond the local Universe are rather poor and only a few examples have been reported (see Comastri ${ }^{4}$ for a review), hereafter I will consider only the effects of two representative values (100 and $400 \mathrm{keV}$ ) for the high energy cut-off. It is possible to account for the resolved fraction of the $1-10 \mathrm{keV}$ background as measured by Chandra ${ }^{19}$ and XMM-Newton ${ }^{22}$ with an appropriate, but reasonable, tuning of the absorption distribution. More specifically the ratio between Compton thin and unabsorbed AGN (assuming $\log N_{H}<22$ as the dividing line) is in the range 2.0-2.4 (for $E_{c u t}=400$ and $100 \mathrm{keV}$ respectively) to be compared with 2.8 in the absorption model of Comastri et al. (1995). The resulting model spectra are reported in Fig. 1 along with a compilation of XRB data which clearly demonstrates a mismatch between the first HEAO1-A2 measurement and the recent estimates in the 2-10 keV band. The maximum deviation is of the order of $40 \%$ at $10 \mathrm{keV}$. For the purposes of the present discussion I consider the analytical fit of Gruber (1992) renormalized upward by a factor 1.3 and slightly modified above about $60 \mathrm{keV}$ to match the HEAO1-A4 MED spectrum. This approximation results in a quite good description of the $2-10 \mathrm{keV}$ XMM-Newton data and settles in between BeppoSAX and Rossi-XTE observations. Though the renormalized Gruber analytical fit provides a reasonable description of the broad band XRB spectrum a few words of caution are appropriate. A closer look at the XRB data points above the peak indicates, besides cross-calibration uncertainties between the A4 LED/MED experiments, a sharp break at $E>$ $40 \mathrm{keV}$ which is responsible for the knee in the analytical approximation. Moreover while it is obvious that the peak in the XRB spectrum must be around a few tens of $\mathrm{keV}$, the exact location and intensity may still be subject to significant uncertainties, especially after the recent measurements below $10 \mathrm{keV}$ which cast doubts on the normalization of the HEAO1-A2 


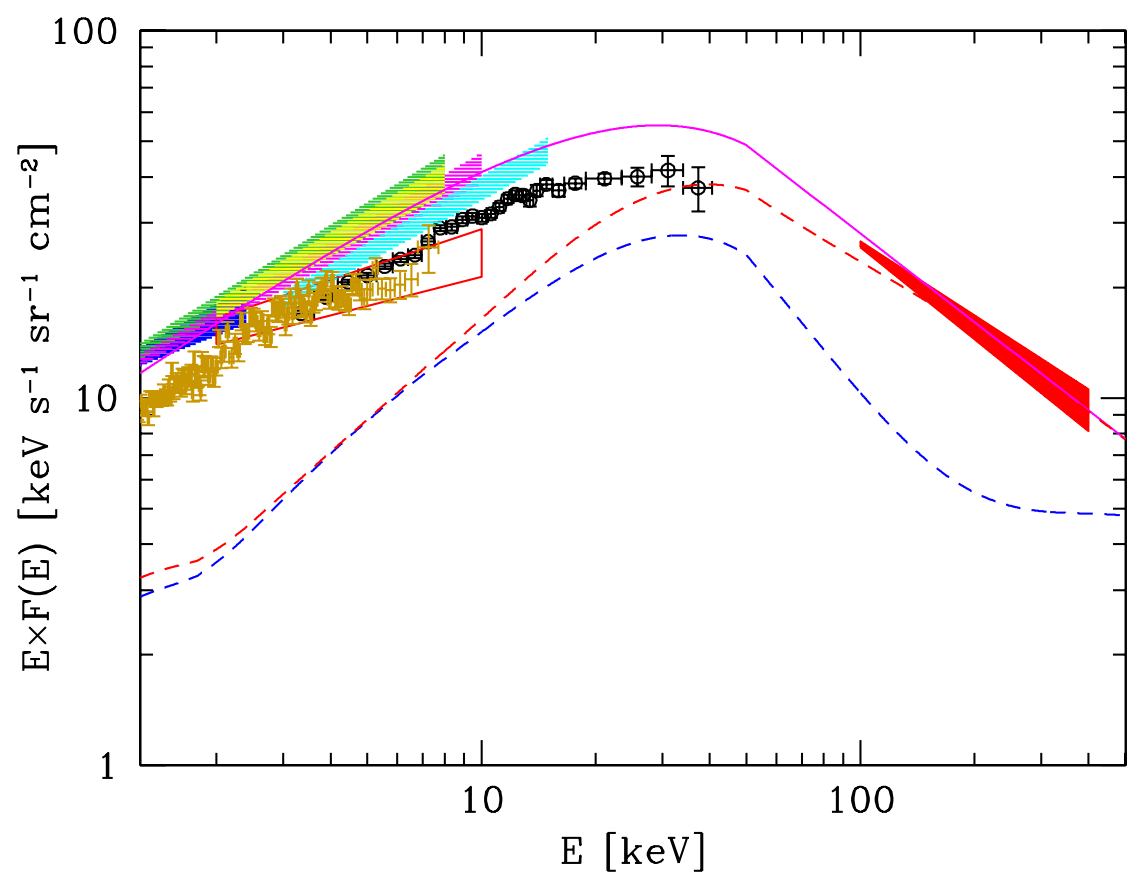

Figure 2. The residual background spectrum after subtraction from the two models reported in Fig. 1 (red line for $E_{\text {cut }}=100 \mathrm{keV}$, blue line for $E_{\text {cut }}=400 \mathrm{keV}$ ). Data as in Figure 1. The HEAO1-A4 LED dataset (green points in Fig. 1) are not reported for clarity.

data. The residual spectra (Fig. 2), computed subtracting the model predictions from the renormalized "observed" XRB intensity, are subjected to the caveats illustrated above.

\section{Conclusions}

Not surprisingly, the shape of the model dependent residual background is reminiscent of that of extremely obscured sources. While the contribution 
of a yet uncovered population of high redshift Compton thick sources ${ }^{6}$ may contribute to fill the gap, it is interesting to note that the residual spectrum peaks at energies of the order of $30-50 \mathrm{keV}$ (depending upon the assumed $\left.E_{c u t}\right)$. If the peak is associated to a characteristic energy in the source spectra, then for a typical redshift of $\simeq 1$ this would correspond to $60-100$ $\mathrm{keV}$ rest-frame. These energies cannot be obtained increasing absorption because at high column densities Compton down-scattering strongly depresses the entire high energy spectrum. An alternative possibility implies high energy cut-off values clustered within a relatively narrow range which in turns depends from the average redshift $\left(E_{\text {cut }} \sim 50-100 \mathrm{keV}\right.$ for $\left.\langle z\rangle \simeq 1\right)$ of the sources. Such a population has to be relatively well localized in space in order to reproduce the rather sharp spectral break of the residual spectrum (especially pronounced if $E_{c u t}=400 \mathrm{keV}$, blue curve in Fig. 2) which otherwise would be smeared out integrating over the cosmological volume.

Taking at the face value the results above described it is also possible that the sources of the residual background are characterized by a truly flat spectrum $(\Gamma \simeq 1.1-1.2)$ up to several tens of $\mathrm{keV}$ which breaks to a much steeper $(\Gamma \simeq 2.6-2.9)$ slope (not necessary an exponential cut-off) at higher energies. Although it seems premature to invoke a new population of sources with a peculiar hard X-ray spectrum the present exercise highlights the need for X-ray observations in the $E>10 \mathrm{keV}$ domain.

\section{Acknowledgments}

I kindly acknowledge support by INAOE, Mexico, during the 2003 Guillermo-Haro Workshop where part of this work was performed. Partial support from ASI I/R/057/02 and MIUR Cofin-03-02-23 grants is also acknowledged. Giancarlo Setti, Gianni Zamorani and Cristian Vignali are acknowledged for a careful reading of the manuscripts and illuminating

comments. A special thank to Raul P.M. Mujica and Roberto P. Maiolino for organizing a stimulating and exciting workshop and for their patience.

\section{References}

1. A.J. Barger et al., A.J. 126, 632 (2003).

2. W.N. Brandt, D.M. Alexander, F.E. Bauer, C. Vignali, in Physics of Active Galactic Nuclei at All Scales, eds. Alloin D., Johnson R., Lira P. (SpringerVerlag, Berlin) astro-ph/0403646

3. A. Comastri, G. Setti, G. Zamorani, G. Hasinger A.EA. 296, 1 (1995).

4. A. Comastri, Review paper for the book "Supermassive Black Holes in the 
Distant Universe", Ed. A. J. Barger, Kluwer Academic, in press, astroph/0403693 (2004).

5. A. De Luca, S. Molendi A.EAA. 419, 837 (2004).

6. A.C. Fabian, R.J. Wilman \& C.S. Crawford M.N.R.A.S. 329, L18 (2002).

7. I. Georgantopoulos, G.C. Stewart, T. Shanks, B.J. Boyle, R.E. Griffiths M.N.R.A.S. 280, 276 (1996).

8. R. Giacconi, J. Bechtold, G. Branduardi et al. Ap.J. 234, L1 (1979).

9. R. Gilli, M. Salvati and G. Hasinger M.N.R.A.S. 324, 757 (2001).

10. D.E. Gruber in "The X-ray background" Eds. X. Barcons, A.C. Fabian, Cambridge University Press, p. 44 (1992).

11. R.L. Kinzer, G.V. Jung, D.E. Gruber, J.L. Matteson, L.E. Peterson Ap.J. 475, 361 (1997).

12. D.H. Lumb, R.S. Warwick, M. Page, A. De Luca A.\&A. 389, 93 (2002).

13. P. Madau, G. Ghisellini and A.C. Fabian M.N.R.A.S. 270, L17 (1994).

14. G.C. Perola, G. Matt, M. Cappi et al. A.ESA. 389, 802 (2002).

15. M. Revnivtsev, M. Gilfanov, R. Sunyaev, K. Jahoda, C. Markwardt A.EA. 411, 329 (2003).

16. G. Risaliti, R. Maiolino, M. Salvati Ap.J. 522, 157 (1999)

17. G.P. Szokoly et al., Ap.J.S.S. , submitted, astro-ph/0312324 (2004).

18. G. Setti, L. Woltjer A.ESA. 224, L21 (1989).

19. P. Tozzi et al. Ap.J. 562, 42 (2001).

20. Y. Ueda, M. Akiyama, K. Otha, T. Miyaji Ap.J. 598, 886 (2003).

21. A. Vecchi, S. Molendi, M. Guainazzi, F. Fiore, A.N. Parmar A.EAA. 349, L73 (1999).

22. M.A. Worsley, A.C. Fabian, X. Barcons, S. Mateos, G. Hasinger, H. Brunner M.N.R.A.S. , in press, astro-ph/0404273 (2004). 